\title{
Explaining trans-phase potential differences with membrane theory, association-induction hypothesis and murburn concept
}

\author{
Hirohisa Tamagawa • Titus Mulembo • \\ Bernard Delalande • Kelath Murali \\ Manoj
}

Received: DD Month YEAR / Accepted: DD Month YEAR

\begin{abstract}
The characteristics of the experimentally measured transmembrane potential (TMP) generated across an artificial membrane intervening two $\mathrm{KCl}$ solutions were found to be explicable using simple principles of electrochemistry, as given within the context of Association Induction Hypothesis (AIH). AIH suggests that the heterogeneous ion distribution which is caused by the adsorption of a mobile ion onto an immobile phase (bearing charge opposite to that of the mobile ion) is responsible for the TMP generation. Therefore, this work proposes AIH could be an important foundation for explaining the origin of TMP. Our experimental observation of nonzero TMP across an electrically charged non-biological/synthetic membrane is found to be intriguing, as such outcomes are classically associated to ion-pumping activities of membrane proteins in a living matter. Another experimental observation of nonzero potential across a neutral membrane is even more intriguing.
\end{abstract}

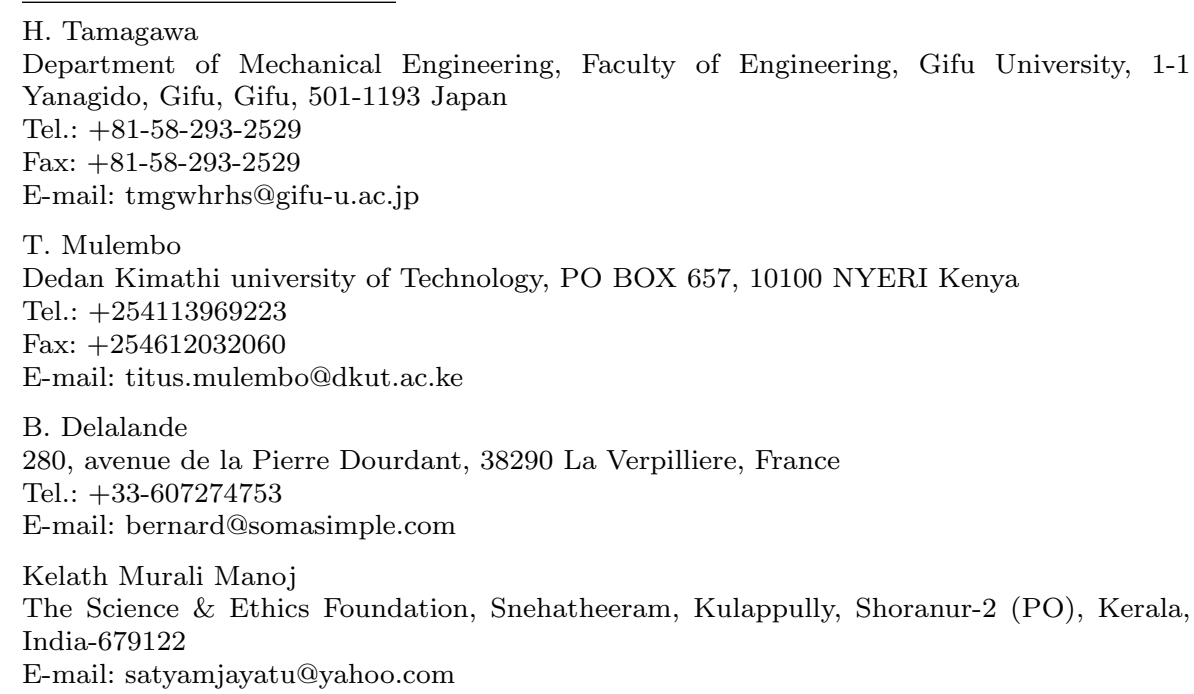


Such a potential behavior is more in harmony with murburn concept, a new proposal for explaining redox metabolic and physiological phenomena.

Keywords trans-membrane potential $\cdot$ membrane theory $\cdot$ associationinduction hypothesis $(\mathrm{AIH}) \cdot$ ion adsorption $\cdot$ murburn concept

\section{Introduction}

A living cell consists primarily of water [1-3]. This watery matter, the living cell, performs biological functions and exhibits unique characteristics. One such distinctive characteristic is the trans-membrane potential (TMP) generation $[4,5]$. Membrane theory states that ion transport across the plasma membrane causes the TMP $[4,5]$. This ion transport is attributed to the sodium-potassium pump, which functions as long as a cell is in a living state. Also, it is broadly accepted that the activity of the sodium-potassium pump consumes ATP [4, $6-9]$. Therefore, the membrane theory posits that living cells intelligently and actively transport ions across the plasma membrane even against their concentration gradients.

Analysis of the TMP has a long history. The pioneering experiments and modeling in the field were done by Hodgkin and Huxley [10-15]. They theorized the dynamic facets of the TMP and laid down the foundations of modern electrophysiology. Their theory could explain the functioning of the membrane pores/channels, which were discovered much later $[11,16]$. Membrane theory is the culmination of the pursuits of Hodgkin-Huxley and other researchers, who have pursued it for more than half a century $[4,7,17]$.

We have recently listed out some experimental observations that are in conflict with the membrane theory. For example: while the prevailing TMP generation mechanism is valid only if the membrane is semi-permeable, even impermeable membranes display TMP $[18,19]$. Also, these experimental traces are quite similar to the computed potentials based on the prevailing membrane theory $[20,21]$. How can this issue be addressed? We believe that this can be settled by thinking beyond the current membrane theory. The AssociationInduction Hypothesis (AIH) is an appealing candidate with respect to inanimate model of the cell $[4,7,17]$. According to AIH, TMP generation could be a consequence of the heterogeneous charge distribution in an electrolytic solution. Since the cell is an assembly of charged matter such as proteins and ions, the potential generated in such a system must obey the laws of physical chemistry regardless of its state, whether living or nonliving [22-24]. The charges in the cell system distribute heterogeneously and abide by the mass action law, Boltzmann distribution, etc. Hence, the charge disparity that is generated in the cell system could contribute to the TMP that we have known in the physical sciences all along. This line of thought raises some important questions: Since ion transporters are not needed for generating the TMP, can there be a simpler explanation for the origin of TMP explained in cells? If trans-membrane ion pumps are needed to maintain ionic differential and TMP, then what will happen when the ion transport ceases? Since active ion 
transport would not be expected in a dead cell, the ionic disparity between the cell-interior and the cell-exterior must be nullified over time, resulting in a total loss of TMP. However, it was reported that the heterogeneous ion distribution (and consequentially, TMP) does not disappear even in dead cells [4, 6]. It is also known that actively respiring mitochondria or photoactive chloroplasts have different TMPs from that of the impulse conducting neurons [25, 26]. The physico-chemical outcomes within the redox metabolic processes in such systems were recently explained with murburn concept [27-29] as an interactive equilibrium of discretized or ordered molecules, ions and radicals. It was suggested that homeostasis and trans-membrane potential of such systems were interconnected phenomena. This implies that redox metabolic processes involving electron-transfer process at the interface also contribute to TMP. Therefore, simple non-living reductionist systems are studied in the light of the developments above and the basic aspects of TMP formation are modeled in such experimental systems.

\section{Experimental}

The composition of various experimental system components were as follows:

\subsection{Potentials in AIH purview}

\subsubsection{Preparation of the connecting/intervening phases}

Gels Three types of hydrogels were prepared in the study: cationic (C-gel), anionic (A-gel) and neutral (N-gel). To $50 \mathrm{ml}$ of deionized water, monomers, crosslinker (N,N'metylenebisacrylamide, $0.154 \mathrm{~g}$ ), polymerization accelerator $\left(\mathrm{N}, \mathrm{N}, \mathrm{N}^{\prime}, \mathrm{N}^{\prime}\right.$ tetramethylethyldiamine, $\left.0.005 \mathrm{~g}\right)$ and polymerization initiator (ammonium persulfate, $0.04 \mathrm{~g}$ ) were added in a systematic and homogeneous manner. Monomers used for the synthesis of C-gel, A-gel and N-gel were "acrylamide $(4.26 \mathrm{~g})$ and allylaminehydrochloride $(1.80 \mathrm{~g})$ ", "acrylamide $(4.26 \mathrm{~g})$ and acrylic acid (144g)" and "acrylamide (5.76g)", respectively. The resulting pregel solutions were heated in $65{ }^{\circ} \mathrm{C}$ water bath for an hour. The gels obtained were separately submerged in deionized water until full swelling. Subsequently, the gels were separately submerged in ethanol to expel nonpolymerized residuals. This $\mathrm{DW} / \mathrm{EtOH}$ washing process was repeated to give fully stable/consistent and well-characterized hydrogels.

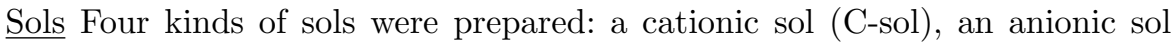
(A-sol), and two neutral sols (N-sol and P-sol). C-sol was prepared simply by heating the $\mathrm{C}$-gel ingredients except crosslinker in $65{ }^{\circ} \mathrm{C}$ water bath for 1 hour. After completion of the sol synthesis, a large quantity of ethanol was mixed with this C-sol. The C-sol was precipitated, retrieved, and mixed again with deionized water. Ethanol was added, resulting in precipitated C-sol. By this 
EtOH/DW solvent exchange, C-sol was washed. Then, C-sol was fully dried in the oven. This fully dried C-sol was mixed with deionized water in the weight ratio of 1:4. The A-sol and $\mathrm{N}$-sol were prepared using the same procedure employed for the preparation of C-sol by starting with the corresponding ingredients of A-gel and $\mathrm{N}$-gel (minus crosslinker). P-sol was prepared by mixing PVA powder and deionized water by the weight ratio of 5:6.

\subsubsection{Preparation of the electrolyte solution}

Various concentrations of $\mathrm{KCl}$ solutions were prepared by the following procedure: $1 \mathrm{M} \mathrm{KCl}$ solution was prepared by dissolving solid $\mathrm{KCl}$ into deionized water. This $1 \mathrm{M} \mathrm{KCl}$ stock solution was serially or appropriately diluted to derive the desired working concentration, $1 \mathrm{M} \sim 10^{-5} \mathrm{M}$.

\subsubsection{Potential measurement}

We made measurements of three types of potentials, as given below.

Surface potential: The experimental setup for the measurement of the surface potential is illustrated in Fig. 1. The potential of the hydrogel surface in reference to the bathing solution is defined as the surface potential.

Prior to measuring the hydrogel surface potential, the hydrogels were equilibrated in the $\mathrm{KCl}$ solution. For example, a piece of $\mathrm{C}$-gel was placed in a $10^{-5} \mathrm{M} \mathrm{KCl}$ solution. After 24 hours, this bathing solution of the C-gel was replaced with a newly prepared $10^{-5} \mathrm{M} \mathrm{KCl}$ solution. This bathing solution exchange process was repeated for five days in a row. Consequently, the desired equilibrated C-gel is obtained. All the hydrogel specimens were prepared in the same manner.

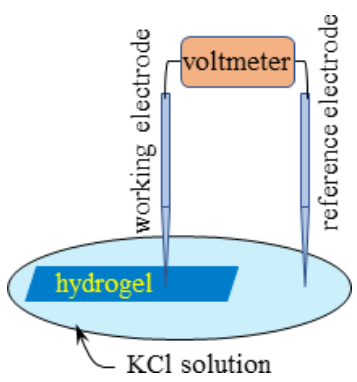

Fig. 1 Setup for the measurement of surface potential

Potential generated across solutions bridged by gels: The potential generated between the two $\mathrm{KCl}$ solutions connected by a hydrrogel was measured using the setup illustrated in Fig. 2, where the Right solution's potential reference is 
$0 \mathrm{mV}$. When making measurements of the TMP using the setups shown in Figs. 2 the "Left solution" is a $10^{-4} \mathrm{M} \mathrm{KCl}$ solution, while the "Right solution" is also a $\mathrm{KCl}$ solution but its concentration ranges from $10^{-5} \mathrm{M}$ to $10^{0} \mathrm{M}$. In the case where the hydrogels (C-gel, A-gel, and N-gel) were used as membranes, these hydrogels were first equilibrated in deionized water. For example, a piece of Cgel was placed in deionized water. After 24 hours, the deionized water bathing the C-gel was replaced with the fresh deionized water. This bathing solution exchange process was repeated for five days in a row. Consequently, the C-gel equilibrated in deionized water is obtained. A-gel and N-gel underwent the same treatment prior to their use.

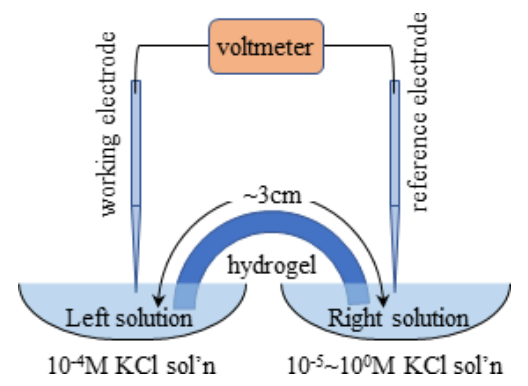

Fig. 2 Setup for the measurement of TMP when using a hydrogel

TMP measurement: In the case where the sol was used as a membrane, six types of sol membranes illustrated in Fig. 3 along with their notations were used. 


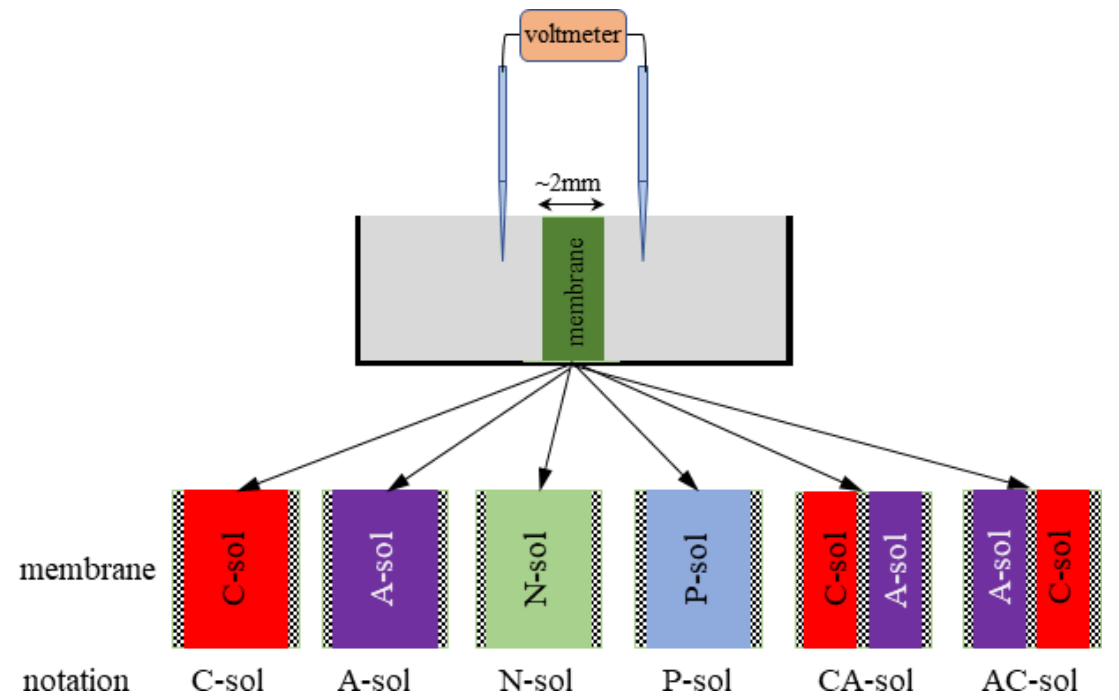

Fig. 3 Structure of membranes and their notations Checkered pattern represents the dialysis membrane where the dialysis membrane is impermeable to the polymer of sol, while it is permeable to the electrolytic solution.

2.2 Potentials predicted by murburn concept

We performed the following experiment to see the enzymatically induced TMP, which is predicted in murburn concept purview.

\subsubsection{Preparation of the connecting/intervening phases}

Figure 4 is a setup for the measurement of enzymatically induced TMP. Although its structure is the same as the setup of Fig. 3, the dialysis membrane bears no immobile charges unlike sols used in Fig. 3 . 


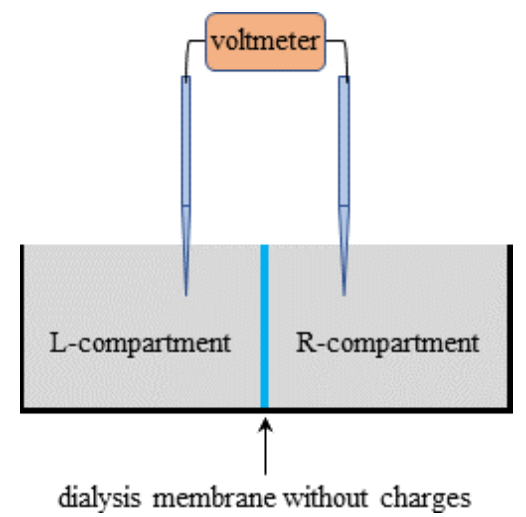

Fig. 4 Setup for the measurement of TMP dynamics

\subsubsection{Preparation of the electrolyte solution}

Two solutions, Sol- $\alpha$ and Sol- $\beta$, were prepared: Sol- $\alpha$ is a dilute $\mathrm{H}_{2} \mathrm{O}_{2}$, which was prepared by diluting $30 \mathrm{wt} \% \mathrm{H}_{2} \mathrm{O}_{2}$ using $0.1 \mathrm{M}$ phosphate buffer $(p H=7.0)$ by a factor of 200 . Sol- $\beta$ is $0.01 \mathrm{M}$ N,N,N',N'-Tetramethyl-1,4phenylenediamine Dihydrochloride (TMPD), which was prepared by dissolving TMPD into 0.1 M phosphate buffer $(p H=7.0) .200 \mu \mathrm{L}$ Sol- $\alpha$ and $200 \mu \mathrm{L}$ Sol- $\beta$ were mixed with $12 \mathrm{~mL}$ deionized water. Another solution, $23 \mu \mathrm{M}$ peroxidase solution, was prepared by dissolving peroxidase into $0.1 \mathrm{M}$ phosphate buffer $(p H=7.0)$.

\subsubsection{Potential measurement}

A half of the mixture prepapred in the section 2.2 .2 was poured into Lcompartment of the experimental setup, while the rest was poured into Rcompartment. TMP was measured with the addition of $100 \mu \mathrm{L}$ of the prepared peroxidase solution to the L-compartment. For serving as a control, TMP was also measured while $100 \mu \mathrm{L}$ water was added.

\section{Results and Discussion}

\subsection{Source of TMP in AIH perspective}

Unlike membrane theory, AIH attributes the origin of TMP to ion adsorptiondesorption. It is explained by graphic expression as below.

First, we shall deal with the AIH explanation for TMP generation. Consider a negatively charged plate, in the electrolytic solution system, as illustrated in Fig. 5. Some mobile cations adsorb on and gather around the plate surface in accordance with law of mass action and Boltzmann distribution. Under such 
a scenario, AIH (which is in line with the framework of electromagnetism) predicts the generation of a non-zero potential in the heterophasic system. Within this context, we investigated the roles of ion adsorption/desorption on TMP, in an experimental system of $\mathrm{KCl}$ solutions separated by various membranes.

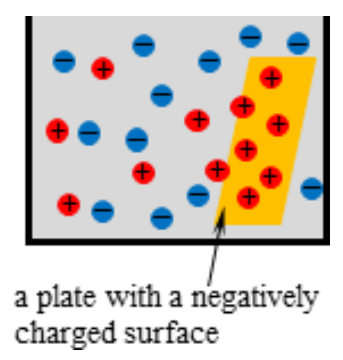

Fig. 5 Negatively charged plate in an electrolytic solution adsorbs positively charged ions from the bulk liquid phase.

\subsubsection{Surface potential}

Imagine a cationic polymer (permeable to electrolytes) in contact with a $\mathrm{KCl}$ solution, as illustrated in Fig. 6. Ions further away from the interfacial region are not illustrated, so as not to complicate illustrations. Therefore, Fig. 6 may not be stoichiometrically accurate. Surface potentials of these cationic polymers $\left(\phi_{s}^{C}\right.$, in reference to the potential at bulk phase of $\mathrm{KCl}$ solution) depends on $\mathrm{KCl}$ concentration. Dashed lines in Fig. 6 represent the expected potential profile. Bulk phase potential is defined as $0 \mathrm{mV}$. Anions adsorbed on the cationic polymer surface neutralize the positive surface charges, resulting in the lowering of surface potential. Increase in $\mathrm{KCl}$ concentration promotes the adsorption of anions on the cationic polymer surface, and it is expected to further reduce the surface potential as illustrated in Fig. $6(\mathrm{a}) \sim(\mathrm{c})$. Therefore, the relationship of the surface potentials of $\phi_{s L}^{C}, \phi_{s M}^{C}$, and $\phi_{s H}^{C}$ (whose definitions are given in Fig. 6) is

$$
\phi_{s L}^{C}>\phi_{s M}^{C}>\phi_{s H}^{C}
$$

\subsubsection{Deriving TMP as a function of surface potentials}

Imagine a system consisting of two $\mathrm{KCl}$ solutions separated by a cationic polymer, which is permeable to electrolytic solution, as illustrated in Fig. 7(a). Now, we theoretically model the TMP of this system. TMP of this system given by the potential at the bulk phase of left $\mathrm{KCl}$ solution in reference to that of right $\mathrm{KCl}$ solution. The system is hypothetically divided into two as shown in Fig. 7(b) and they are denoted by Left Region and Right Region, respectively. 


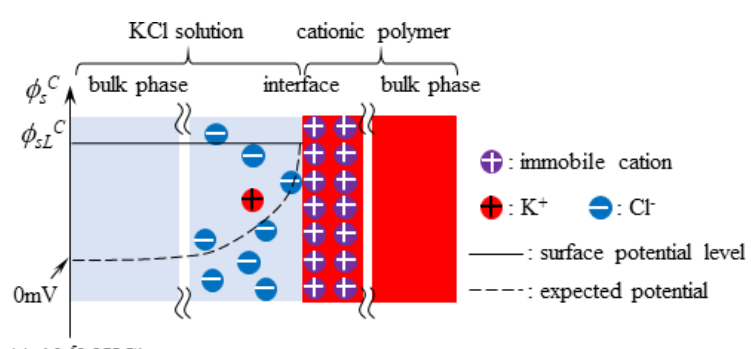

(a) $10^{-5} \mathrm{M} \mathrm{KCl}$

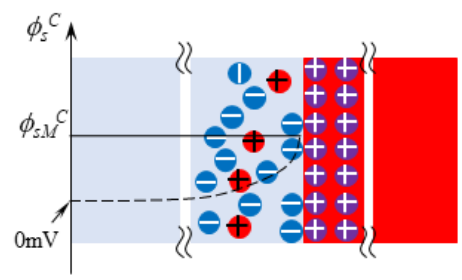

(b) $10^{-2} \mathrm{M} \mathrm{KCl}$

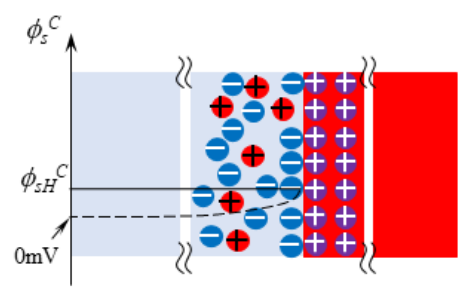

(c) $10^{\circ} \mathrm{M} \mathrm{KCl}$

Fig. 6 A cationic polymer, which is permeable to electorlytic solution, in contact with a $\mathrm{KCl}$ solution $\mathrm{KCl}$ concentrations are (a) $10^{-5} \mathrm{M}$, (b) $10^{-2} \mathrm{M}$ and (c) $10^{0} \mathrm{M}$. Macroscopic electroneutrality holds in the both phases of $\mathrm{KCl}$ soluton and cationic polymer and the potential in the $\mathrm{KCl}$ solution bulk phase is defined $0 \mathrm{mV}$.

These two Regions are individually identical to the system shown in Fig. 6 . The potential represented by the dashed lines in Fig. 7(b) is expected to be generated because of the ion adsorption on the cationic polymer surface as discussed earlier. $\phi_{s \ell}^{C}$ and $\phi_{s h}^{C}$ shown in Fig. 7(b) are surface potential at the left and right cationic polymer surfaces, respectively. Both Left Region and Right Region are combined into one again, and ideally $\phi_{s \ell}^{C}$ level is same as $\phi_{s h}^{C}$ level as shown in Fig. 7(c). Therefore, the TMP, $\Delta \phi_{m}^{C}$, could be calculate using these surface potentials as given by Eq. 2 after redefining the $0 \mathrm{mV}$ reference potential as shown in Fig. 7(c) as long as AIH is valid.

$$
\Delta \phi_{m}^{C}=\left(-\phi_{s \ell}^{C}\right)+\phi_{s h}^{C}=\phi_{s h}^{C}-\phi_{s \ell}^{C}
$$



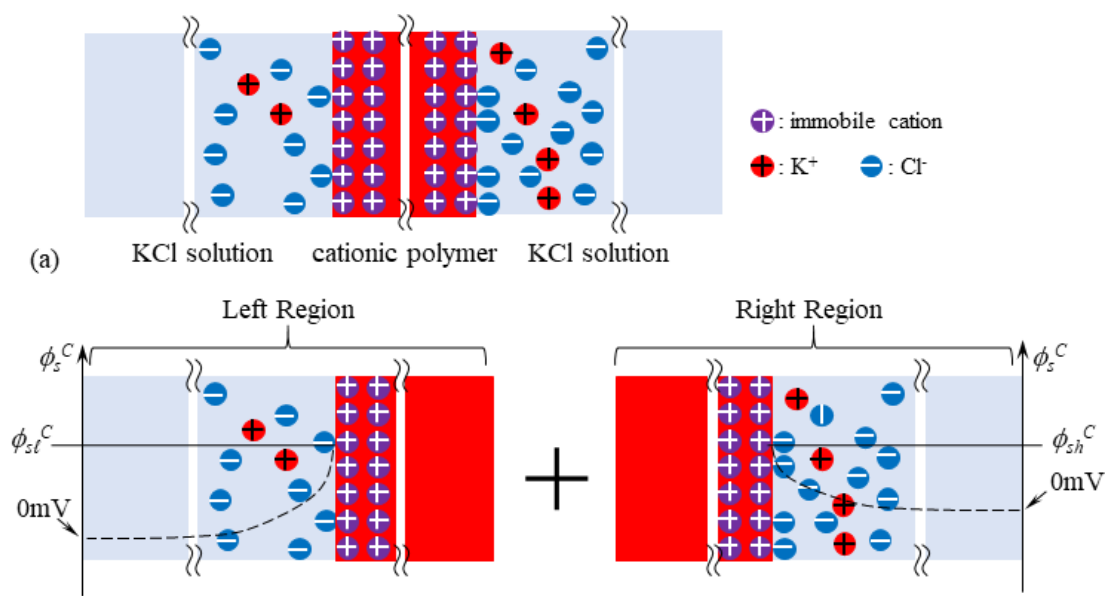

(b)

_ : surface potential level

$--\cdot$ : expected potential

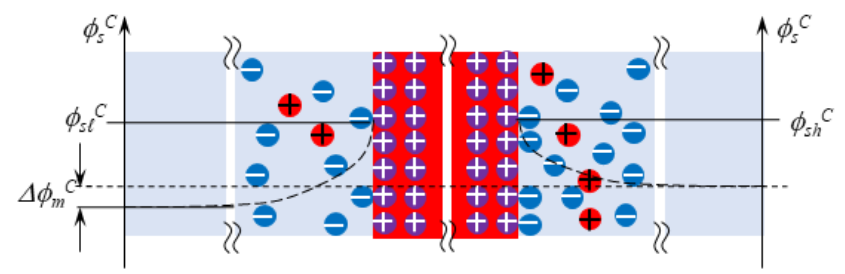

(c) $\cdots \cdots$ redefined $0 \mathrm{mV}$ reference potential level common for whole system

Fig. 7 (a) Two $\mathrm{KCl}$ solutions separated by cationic polymer which is permeable to electrolytic solution, (b) Hypothetically divided system shown in (a), $\quad$ (c) TMP, $\Delta \phi_{m}^{C}$, expected to be generated in the system shown in (a)

\subsubsection{TMP across a combinatorial or heterologous (mixed) membrane}

Imagine a system consisting of two $\mathrm{KCl}$ solutions separated by a combinatorial or mixed membrane composed of an anionic polymer and a cationic polymer, as illustrated in Fig. 8(a) where both polymers are permeable to electrolytic solution. This system is hypothetically divided into two, as shown in Fig. 8(b). These two systems can be individually considered. The Left Region in Fig. 8(b) is obtained simply by replacing the cationic polymer in Fig. 6 by an anionic polymer. Unlike the system in Fig. 6, cations are adsorbed on the anionic polymer surface. The potential represented by the dashed lines in Fig. 8(b) is expected to be generated because of the ion adsorption on the anionic polymer surface and cationic polymer surface. $\phi_{s \ell}^{A}$ and $\phi_{s h}^{C}$ shown in Fig. 8(b) are surface potential of anionic polymer and surface potential of the cationic polymer, respectively. Therefore, the TMP, $\Delta \phi_{m}^{A C}$, could be calculated using these surface potentials as given by Eq. 3 after redefining the $0 \mathrm{mV}$ reference potential as shown in Fig. 8(c) as long as AIH is valid, where $\Phi$ in Fig. 8(c) is 
the interfacial potential generated at the boundary between the anionic and the cationic polymers.

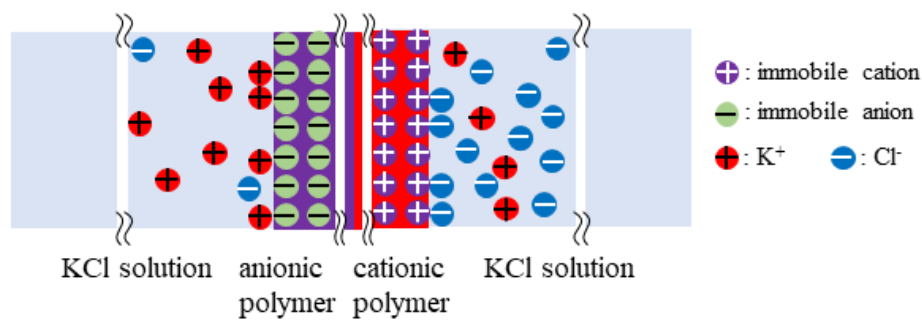

(a)
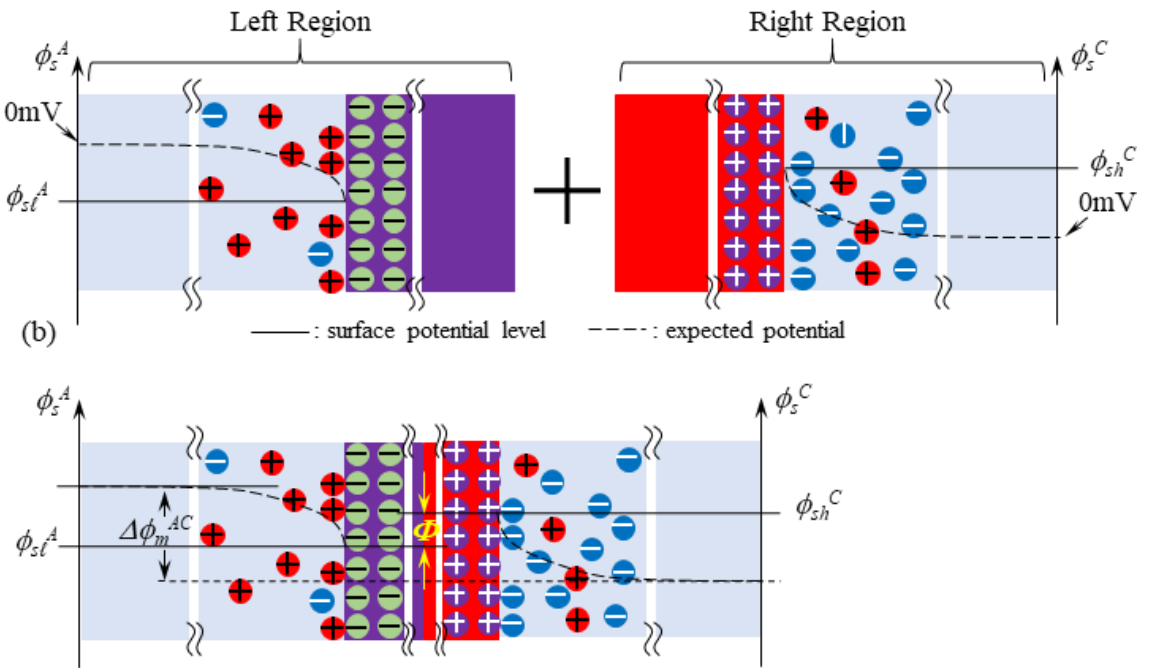

(c) -....- redefined $0 \mathrm{mV}$ reference potential level common for whole system

Fig. 8 (a) Two $\mathrm{KCl}$ solutions separated by a combinatorial membrane composed of an anionic polymer and a cationic polymer which are both permeable to electrolytic solution,

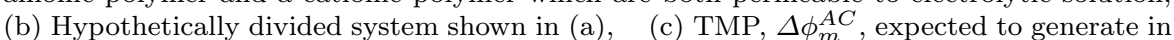
the system shown in (a) where $\Phi$ represents the potential generated at the interface between the anionic and cationic polymers.

$$
\Delta \phi_{m}^{A C}=\left(-\phi_{s \ell}^{A}\right)+\Phi+\phi_{s h}^{C}=\phi_{s h}^{C}+\Phi-\phi_{s \ell}^{A}
$$

AIH suggests that the TMP is the summation of potentials generated across individual regions of the system in question, and these potentials are generated independently of one another. Therefore, the following noticeable prediction is derived. Eq. 2 is for the experimental system shown in Fig. 7, while Eq. 3 is for the experimental system shown in Fig. 8. What is common for both systems are (i) $\mathrm{KCl}$ concentration in the left solution is constant, while that in the right solution $\mathrm{KCl}$ concentration varies and (ii) the Right Region for both 
experimental systems of Figs. 7 and 8 are structurally and chemically identical to each other. Therefore, despite the expectation of clear difference between the TMP $\left(\Delta \phi_{m}^{C}\right)$ for the system Fig. 7 and the TMP $\left(\Delta \phi_{m}^{A C}\right)$ for the system Fig. 8, their difference $\Delta \phi_{m}^{C}{ }_{-} A C$ given by Eq. 4 is maintained constant. This is indicated by Eq. 5 as long as the concentration of the right $\mathrm{KCl}$ solution is same for both experimental systems each other. Here, $\phi_{s \ell}^{A}$ and $\phi_{s \ell}^{C}$ are constant, since the concentration of left $\mathrm{KCl}$ solution is maintained constant at $10^{-4} \mathrm{M}$, and $\Phi$ is constant, too, since the anionic polymer-cationic polymer interface is not contact with the right $\mathrm{KCl}$ solution whose concentration is varied. So, if Fig. 6 is confirmed, it is a strong indication of AIH validity.

$$
\begin{gathered}
\Delta \phi_{m}^{C_{-} A C}=\Delta \phi_{m}^{C}-\Delta \phi_{m}^{A C} \\
\Delta \phi_{m}^{C_{-} A C}=\Delta \phi_{m}^{C}-\Delta \phi_{m}^{A C}=\left(\phi_{s h}^{C}-\phi_{s \ell}^{C}\right)-\left(\phi_{s h}^{C}+\Phi-\phi_{s \ell}^{A}\right) \\
=\phi_{s \ell}^{A}+\Phi-\phi_{s \ell}^{C}=\text { const. }
\end{gathered}
$$

3.2 Experimental analysis of surface potential and TMP

\subsubsection{Gel surface potential}

In the section 3.1.1, the cationic polymer surface potential was theoretically treated within the frame work of $\mathrm{AIH}$, and it was predicted that the potential decreases by the concentration increase of $\mathrm{KCl}$ bathing solution. Fig. 9 shows the C-gel surface potential experimentally measured using the setup shown in Fig. 1. it suggests that the experimental C-gel surface potential behavior agrees with theoretical prediction.

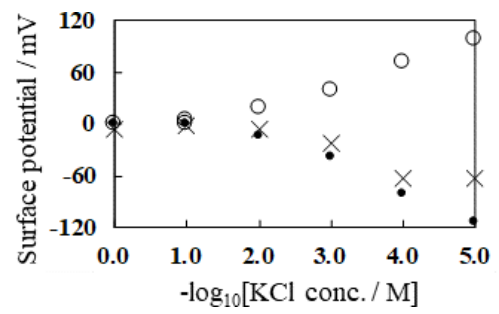

Fig. 9 Experimental surface potential vs. $-\log _{10}[\mathrm{KCl}$ conc./ M $]$ O: C-gel, •: A-gel, $\times$ : N-gel

Since the anionic polymer is negatively charged, cations adsorb on it. Therefore, the opposite potential behavior to the surface potential of cationic polymer is expected theoretically according to the AIH. Namely, the surface potential should go higher with the increase of concentration of $\mathrm{KCl}$ bathing 
solution. Fig. 8 shows that the experimental A-gel surface potential behavior agrees with theoretical prediction.

As N-gel is an electrically neutral gel, ions are not expected to adsorb on it. Therefore, it is expected to exhibit a constant potential regardless of the $\mathrm{KCl}$ concentration. However, contrary to the expectations, the experimental data $($ Fig. 9) $($ symbol $\times$ ) shows a profile similar to that of anionic gel. The issue will be discussed in section 3.4 .

\subsubsection{TMP across a hydrogel}

The potentials across three types of hydrogels were measured using the setup shown in Fig. 2 and the results are shown in Fig. 10. The experimentally measured TMP was analyzed following the discussion given in the section 3.1.2. Plugging the experimentally measured C-gel surface potential shown in Fig. 9 into Eq. 2 results in theoretically calculated TMP across the C-gel, which is shown by symbol " $\times$ " in Fig. 10(a). The same TMP measurement across $\mathrm{A}$-gel and $\mathrm{N}$-gel was performed and the same theoretical calculation was performed, too. The outcomes are shown in Fig. 10(b) and (c). Since the experimentally measured TMPs are in good agreement with the theoretically calculated TMPs in all three gel types, the validity of AIH is strongly indicated.

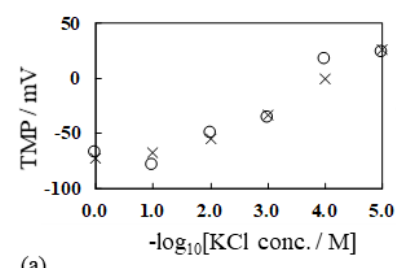

(a)

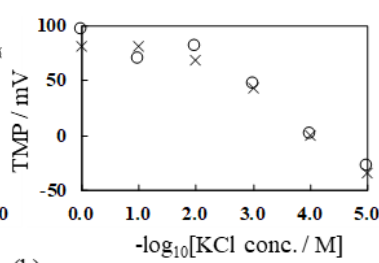

(b)

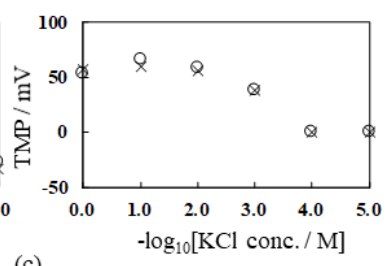

(c)

Fig. 10 TMP vs. $-\log _{10}[$ Right sol'n $\mathrm{KCl}$ conc./ M] ०: Experimentally measured TMP, $\times$ : Theoretically calculated TMP using the experimentally measured surface potentials given in Fig. 9 (a) C-gel, (b) A-gel, (c) N-gel

\subsection{TMP vs. $\mathrm{KCl}$ concentration when a liquid membrane is in use}

Since the plasma membrane bears the characteristics of fluidity like a sol [30, 31 , we studied various types and combinations of such membranes. Measurements for the potential generated across the sol were made using the setup shown in Fig. 3. Figs. 11(a) and(b) show the measured TMPs across the electrolytic sols (C-sol, A-sol, CA-sol, AC-sol) and the neutral sols (N-sol and $\mathrm{P}$-sol), respectively. 


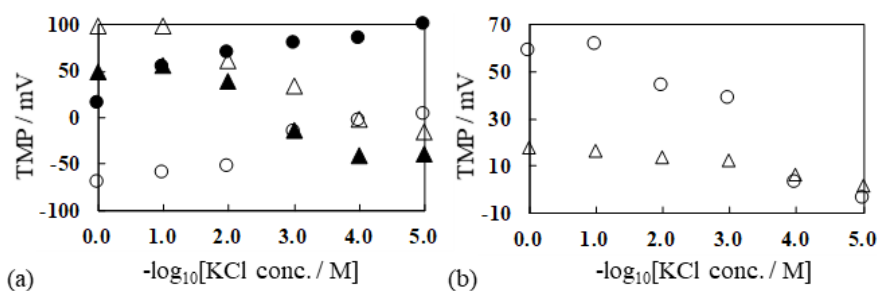

Fig. 11 Experimental TMP vs. - $\log _{10}[$ Right sol'n KCl conc./ M] (a) O: C-sol $\triangle$ : A-sol •: AC-sol $\Delta$ : CA-sol (b) O: N-sol $\triangle$ : P-sol

TMP across AC-sol is different from the TMP across C-sol. However, Eq. 5 suggests that the difference is given by a unique constant value as long as the $\mathrm{KCl}$ concentration of the right solution in contact with AC-sol is same as that in contact with C-sol. The experimentally measured TMPs across AC-sol and C-sol in Fig. 11(a) show that Eq. 5 is basically sufficed. If the AC-sol is flipped horizontally, A-sol surfaces comes to contact with the right $\mathrm{KCl}$ solution. Therefore, the TMP across CA-sol comes to be different from the TMP across A-sol. However, the difference must be given by a unique constant value as well as long as the $\mathrm{KCl}$ concentration of the right solution in contact with CA-sol is same as that in contact with A-sol. The experimentally measured TMP across CA-sol and A-sol shown in Fig. 11 basically agrees with this prediction as expected. These outcomes are proofs for the AIH validity. However, TMP across the neutral sols shown in Fig. 11(b) exhibits the $\mathrm{KCl}$ concentration dependence. It appears to disprove AIH. This issue is discussed in the next section.

\subsection{TMP in neutral matters and further discussion toward Murburn concept}

Figures. 9 and 11(b) shows that the use of electrically neutral membranes (N-gel, N-sol, and P-sol) causes the nonzero potential generation, and these potentials exhibit $\mathrm{KCl}$ concentration dependence. Since the mobile ions cannot adsorb on the electrically neutral matters, AIH suggests that a constant potential should be generated irrespective of the $\mathrm{KCl}$ concentration. Therefore, such results could imply that- (i) there could be 'negative impurities' within the neutral matter or/and (ii) other parameters/dimensions could also be involved. We present a brief discussion on various aspects of this issue.

The oxygen atom of acrylamide (the main component of N-gel and N-sol) or PVA (the main component of $\mathrm{P}$-sol) could come to possess a negative dipole and this could help immobilizing a positive ion like $\mathrm{K}^{+}$within the matrix. If this could result, we can explain the profiles of the neutral gels/sols, which are similar to the anionic matrices. The ability to behave 'non-neutrally' can also come due to acrylamide's hydrolytic interaction with water that leads to the formation of carboxylate [32] or it could also be inherent in the universal solvent of water molecule [33-40]. The dynamic temporal changes of 
TMP measured when an excitable cell is primed with a suitable cue gives rise to an 'action potential' (AP) signature. Considering AP generation (temporal variation of TMP) as a sign of life is a common notion since the activity of the sodium pump accompanies the AP generation according to the membrane theory $[4,7,10]$. However, spontaneous AP-like potential generation in the nonliving system like that of proteinoid spheres of Fox et al. [41-45], which has no ion-transporting pumps, is a classical example. Furthermore about AP analysis, elucidation of nerve signal propagation mechanism is still a central topic in physiology, and even totally new idea has attracted physiologists attention: Heimburg and Jackson proposed a new mechanism of nerve signal propagation $[46,47]$. They attributed the nerve signal propagation to the mechanical wave conduction through the plasma membrane, while it is usually attributed to the electrical wave conduction. They do not deny the electrical potential generation, but they do not attribute the biological fundamentality to the electrical potential wave. Our belief is that current physiology addresses individual research topics by wielding chosen theories which are appropriate only to that topic focused on. Therefore, physiology and its related research fields have been in the state of awaiting a totally new and comprehensive idea.

One of us, K.M.M, has reasoned that redox homeostasis and transmembrane potential are inter-connected phenomena related to electrontransfer equilibriums in the bioenergetic routines of mitochondria and chloroplasts [27-29]. The new perception is enabled by "murburn concept". It was reasoned that TMP is not the driving force of ATP-synthesis, but is rather a consequence of oxygen-centered radical formation in these organelles, resulting due to effective charge separation by membrane-embedded proteins. We have solved the $\mathrm{K}>\mathrm{Na}>\mathrm{Mg}>\mathrm{Ca}$ order of cation distribution in cells, based on the predictability of murburn concept [48]. The murburn model affords a fresh perspective to redox metabolism and electrophysiology, also enabling a new explanation for the physiology of vision [48-50]. Extrapolating those findings to the current experiments could suggest that since our works were done in oxic environment, the diradical could spontaneously sponsor effective charge separation (in conjunction with the matrix material) to give anionic species in milieu and this could potentially account for the TMP trends seen.

TMPs shown in Fig 12 were obtained by performing the experiments described in the section 2.2. Addition of peroxidase in the L-compartment clearly causes the significant TMP fluctuation, while the addition of deionized water induced virtually no change. Murburn concept states this process: Addition of peroxidase must produce a small amount of superoxide. This, in short time, gets neutralized after abstracting electron/hydrogen atom from TMPD, and therefore the potential returns to the baseline. Hence, the dynamics of membrane potential (AP generation) could be explicable in the course of murburn concept, and the validation of murburn concept implicates even the fundamental conceptual reform of metabolic activity such as ATP-synthesis as touched upon earlier. 

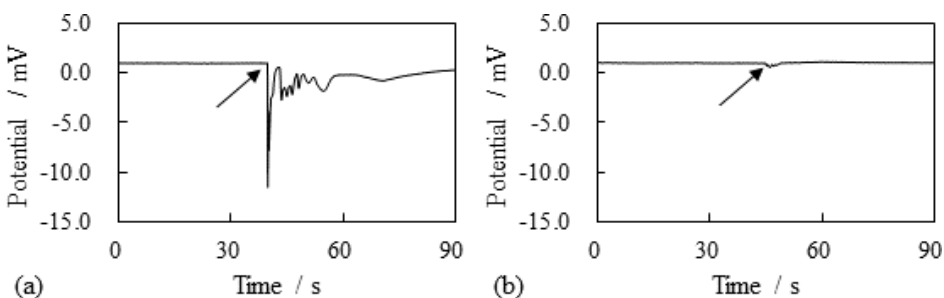

Fig. 12 TMP across a dialysis membrane The arrows indicate the moments (a) $100 \mu \mathrm{L}$ of $0.23 \mu \mathrm{M}$ peroxidase is added in the L-compartment and (b) $100 \mu \mathrm{L}$ of deionized water is added in the L-compartment, respectively.

Figure 13 shows the salient mechanistic aspects for the development of TMP according to the various schools of thought. Herein, we suggest that the AIH-murburn perspective can afford a simple model to understand the origin and simple variations of TMP.

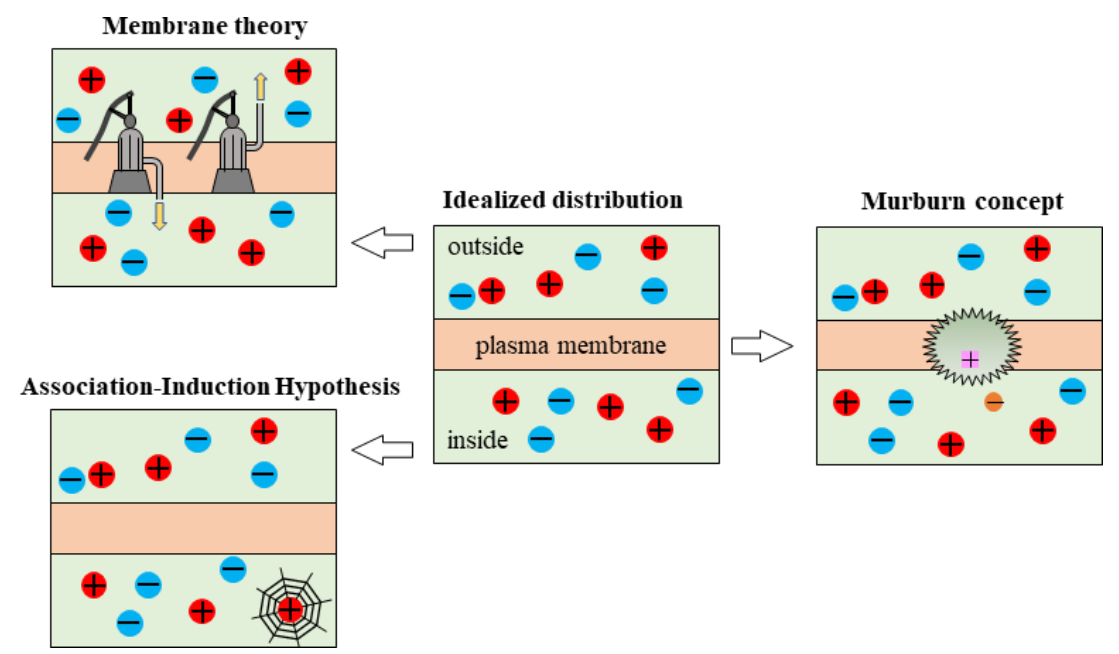

Fig. 13 The various proposals for the explanation of trans-phase or trans-membrane potential (TMP) in physiology.

In the center of Fig. 13, a scheme to represent the inside and outside portions of a cell with the intervening membrane is shown, with both sides being electrically neutral and each phase containing an equivalent amount of three positive and three negative ions. From this resting state, the arrows are made to show the process that could result in the net negative TMP (resting state) usually seen in physiology. In the membrane theory, TMP results owing to membrane-lodged proteins (depicted with two pumps) actively pumping in and out ions (say, proton or sodium ion), leading to an excess positive ion in 
the outside. In the AIH purview, the potential difference results due to the presence of negatively charged adsorbent in the bulk phase (depicted with a spider web), which chelates and removes a positive ion from the inside phase; and this results in an excess negative ion on the inside. In contrast to the two ionic theories above, murburn concept is an electronic theory involving stochastic interactive exchange of electrons between molecules, ions and transiently formed radicals. A transient effective charge separation on any phase (catalyzed by a suitable component, depicted by a thorny component lodged and suspended from the membrane) could lead to the formation of a free electron, which can be taken up by a species like oxygen molecule to give superoxide. This ionic radical, if present in the inside, can lead to a net negative TMP. What our work poses here is: Which is the perspective that best explains the physiological observations? Are all three of the proposals needed?

\section{Conclusions}

Localized charge fixed on the matter in an electrolytic solution inevitably causes mobile ions' adsorption. Consequently, it results in heterogeneous ion distribution, causing the nonzero potential. In the light of the evidence presented herein, AIH should seen as a/the fundamental source/origin of TMP in physiology. It is well-known that proteins present in the bulk-phase or membrane phase could have ample negative charges afforded by carboxylate residues of amino acids. These can aid in the adsorption of positive ions, thereby resulting in the net negative TMP (in versus out) usually seen in cells. While the purely ionic theory of AIH could suffice to explain the gross outcomes in reductionist systems, the biological milieu would also require electrontransfer based approach, as afforded by the new idea of murburn concept. Therefore, we call for an amalgamation of the ideas of AIH, murburn concept and certain aspects of membrane theory (like ion channels and aquaporins). Such an approach could afford a simpler explanation for cation distribution in cells and the dynamics of homeostasis/TMP/AP in cells or organelles.

Funding The authors received no specific financial support for this work.

Conflicts of interest The authors declare no conflicts of interest.

\section{References}

1. G. N. Ling, The physical state of water in living cell and model sytems. Annals New York Academy of Sciences 1965, 125, 401-417.

2. E. Del Giudice, V. Elia, A. Tedeschi, The Role of Water in the Living Organisms . Neural Network World 2009, 19, 355-360.

3. F. Sachs, M. V. Sivaselvan, Cell volume control in three dimensions: Water movement without solute movement. J. Gen. Physiol. 2015, 145, 373-380.

4. G. Ling, A revolution in the physiology of the living cell. Krieger, Malabar, FL (1992). 
5. G. H. Pollack, Cell electrical properties: reconsidering the origin of the electrical potential. Cell Biology International 2015, 39, 237-242.

6. G. Ling, Maintenance of low sodium and high potassium levels in resting muscle cells. J. Physiol. 1978, 280, 105-123.

7. G. Ling, Life at the Cell and below Cell Level. The Hidden History of a Fundamental Revolution in Biology. Pacific Press, NY (2001)

8. K. L. Ode, T. Katsumata, D. Tone, H. R Ueda, Fast and slow $\mathrm{Ca}^{2+}$-dependent hyperpolarization mechanisms connect membrane potential and sleep homeostasis. Current Opinion in Neurobiology 2017, 44, 212-221.

9. M. Kowacz, G. H. Pollack, Cells in New Light: Ion Concentration, Voltage, and Pressure Gradients across a Hydrogel Membrane. ACS Omega 2020, 5, 21024-21031.

10. I. Tasaki, Physiology and Electrochemitry of Nerve Fibers. Academic Press, NY (1982)

11. J. Cronin, Mathematical Aspects of Hodgkin-Huxley Neural Theory. Cambridge University Press, NY (1987)

12. G. B. Ermentrout, D. H. Terman, D.H., Mathematical Foundations of Neuroscience. Springer, NY (2010)

13. L. Holland L, H. W. de Regt, B. Drukarch, Electrochemical Interpretation of Propagation of the Change in the Membrane Potential Using the Goldman-Hodgkin-Katz Equation. Electroanalysis 2017, 29, 2656 - 2664

14. B. Drukarch, H. A. Holland, M. Velichkov, J. J.G. Geurts, P. Voorn, G. Glasa, H. W. de Regt, Thinking about the nerve impulse: A critical analysis of the electricity centered conception of nerve excitability. Progress in Neurobiology 2018, 169, 172-185.

15. L. Holland L, H. W. de Regt, B. Drukarch, Thinking About the Nerve Impulse: The Prospects for the Development of a Comprehensive Account of Nerve Impulse Propagation. Front. Cell. Neurosci. 2019, 13, doi: 10.3389/fncel.2019.00208

16. R. Keynes, J.Z. and the discovery of squid giant nerve fibers. Journal of Experimental Biology 2005, 208, 179-180.

17. L. Jaeken, The neglected functions of intrinsically disordered proteins and the origin of life. Progress in Biophysics and Molecular Biology 2017, 126, 31-46.

18. G. Colacicco, Electrical potential at an oil/water interface. Nature 1965, 207, 936-938.

19. G. Colacicco, Reversal of potential across a liquid non-aqueous membrane with regard to membrane excitability. Nature 1965, 207, :1045-1047.

20. H. Tamagawa, Mathematical expression of membrane potential based on Ling's adsorption theory is approximately the same as the Goldman-Hodgkin-Katz equation. J. Biol. Phys. 2018, 45, 13-30.

21. H. Tamagawa, K. Ikeda, Another interpretation of the Goldman-Hodgkin-Katz equation based on Ling's adsorption theory. European Biophysics J. 2018, 47, 869-879.

22. G. N. Lewis, M. Randall, Thermodynamics. McGraw-Hill, NY (1961)

23. G. M. Barrow, Physical Chemistry. McGraw-Hill, NY (1973)

24. H. Tamagawa, M. Funatani, K. Ikeda, Ling's Adsorption Theory as a Mechanism of Membrane Potential Generation Observed in Both Living and Nonliving Systems. Membranes 2016, 6, 11. https://doi.org/10.3390/membranes6010011

25. A. A. Gerencser, C. Chinopoulos, M. J. Birket, M. Jastroch, C. Vitelli, D. G. Nicholls, M. D. Brand, Quantitative measurement of mitochondrial membrane potential in cultured cells: calcium-induced de- and hyperpolarization of neuronal mitochondria. $J$. Physiol. 2012, 590, 2845-2871.

26. W. Kühlbrandt, Structure and function of mitochondrial membrane protein complexes. BMC Biology 2015, 13, Article\#: 89.

27. K. M. Manoj, Aerobic Respiration: Criticism of the Proton-centric Explanation Involving Rotary Adenosine Triphosphate Synthesis, Chemiosmosis Principle, Proton Pumps and Electron Transport Chain. Biochemistry Insights 2018, 11, 1-23.

28. K. M. Manoj, V. Soman, V. D. Jacob, A. Parashar, D. A. Gideon, M. Kumar, A. Manekkathodi, S. Ramasamy, K. Pakshirajan, N. M. Bazhin, Chemiosmotic and murburn explanations for aerobic respiration: Predictive capabilities, structure-function correlations and chemico-physical logic. Archives of Biochemistry and Biophysics 2019, 676, 108128.

29. K. M. Manoj, A. Manekkathodi, A. Manekkathodi, Light's interaction with pigments in chloroplasts: The murburn perspective. J. Photochem. Photobiol. 2021, 5, 100015. 
30. F. Gutmann, H. Kayzer (EDs), Modern bioelectrochemistry. Plenum Press, NY (1986)

31. M. B. Jackson, Molecular and Cellular Biophysics. Cambridge University Press, Cambridge (2006)

32. T. Tanaka, Gels. Sci. Am. 1982, 244, 124-138.

33. J. K. Gregory, D. C. Clary, K. Liu, M. G. Brown, R. J. SayKally, The Water Dipole Moment in Water Clusters. Science 1997, 275, 814-817.

34. M. Tokmachev, A. L Tchougréeff, R. Dronskowski, Hydrogen-Bond Networks in Water Clusters $\left(\mathrm{H}_{2} \mathrm{O}\right)_{20}$ : An Exhaustive Quantum-Chemical Analysis. ChemPhysChem 2010, 11, 384-388.

35. P. Laurson, U. Mäerog, Water and water clusters in biological systems. Agronomy Research 2015, 13, 1253-1259.

36. J.-M. Zheng, G. H. Pollack, Long-range forces extending from polymer-gel surfaces. Phys. Rev. E 2003, 68, 031408.

37. J.-M. Zheng, W.-C. Chin, E.Khijniak, G. H. Pollack, Phase Transitions in Cell Biology: Unexpected linkage between unstirred layers, exclusion zone, and water. Adv. Colloid Interface Sci. 2006, 127, 19-27.

38. J.-M. Zheng, G. H. Pollack, Water and the Cell: Solute exclusion and potential distribution near hydrophilic surfaces. Springer, Netherlands (2006)

39. G. H. Pollack, J. Clegg, Phase Transitions in Cell Biology: Unexpected linkage between unstirred layers, exclusion zone, and water. Springer, NY (2008)

40. B. Chai, H. Yoo, G. H. Pollack, Effect of Radiant Energy on Near-Surface Water. J. Phys. Chem. B 2009, 113, 13953-13958.

41. Y. Ishima Y, A. T. Przybylski, S. W. Fox, Electrical membrane phenomena in spherules from proteinoid and lecithin. Bio. Systems 1981, 13, 243-251.

42. A. T. Przybylski, W. P. Stratten, R. M. Syren, S. W. Fox, Membrane, action, and oscillatory potentials in simulated protocells. Die Naturwissenschaften 1982, 69, 561-56.

43. A. T. Przybylski, S. W. Fox, Excitable artificial cells of proteinoid. Appl. Biochem. Biotechnol. 1984, 10, 301-307.

44. A. T. Przybylski, Excitable cell made of thermal proteinoids. Biosystems 1985, 17, 281-288.

45. G. Vaughan, A. T. Przybylski, S. W. Fox, Thermal proteinoids as excitability-inducing materials. Biosystems 1987, 20, 219-223.

46. T. Heimburg, A. Jackson, On soliton propagation in biomembranes and nerves. Proceedings of the National Academy of Sciences 2005, 102, 9790-9795.

47. T. Heimburg, A. Jackson, The Brain, Reimagined. Sci. Am. 2018, 318, 60-67.

48. K. M. Manoj, H. Tamagawa, Revisiting the mechanisms for cellular homeostasis and electrophysiological responses: Classical membrane theory, association-induction hypothesis and murburn concept. OSF Preprints 2020. doi: 10.31219/osf.io/e2ynk

49. K. M. Manoj, V. D. Jacob, The murburn precepts for photoreception. Biomedical Reviews 2020, 31, 67-74.

50. K. M. Manoj, Murburn concept: a paradigm shift in cellular metabolism and physiology. Biomolecular Concepts 2020, 11, 7-22. 